\title{
IMPLEMENTATION AND COMPARATIVE EVALUATION OF VARIOUS ARCHITECTURES OF ULTRASONIC PHOTOREFRACTIVE SENSORS
}

\author{
S. de Rossi*1, Ph. Delaye ${ }^{1}$, J.C. Launay ${ }^{2}$, G. Roosen ${ }^{1}$ \\ ${ }^{1}$ Laboratoire Charles Fabry de l'Institut d'Optique, \\ UMR 8501 du CNRS, BP 147, 91403 Orsay Cedex, France. \\ 2Institut de Chimie de la Matière Condensée de Bordeaux, \\ UPR 9048 du CNRS, avenue du Dr A. Schweitzer, 33608 Pessac cedex, France.
}

\begin{abstract}
A novel optical sensor for the detection of ultrasonic motion has been recently developed. It allows to reach $25 \mathrm{pm}$ displacement sensitivity with a large frequency range bandwidth $100 \mathrm{MHz}$. We will present a comparative study between various architectures of this ultrasonic detection system we have implemented. It uses InP:Fe or CdZnTe:V holographic crystals and operates with CW laser at $1.06 \mu \mathrm{m}$. Two configurations called the direct detection and the anisotropic diffraction configurations work with either plane or speckled waves. A third discussed configuration works with depolarized speckled waves. We measured their relative detection limits as a function of the applied electric field that governs photorefractive efficiency of the materials. Experimental results are well described by theory, using photorefractive models relevant to the used crystals.
\end{abstract}

Keywords : Photorefractive effect, ultrasonic detection, screening field.

\section{Presentation of various architectures}

Phase demodulation is based on a two wave mixing (TWM) mechanism. A rapidly and weakly phase modulated signal beam and a pump beam interfere in a photorefractive crystal. As the period of the phase modulation is much smaller than the response time of the crystal, both waves write an average hologram. The pump beam diffracts on it to give an unmodulated local oscillator that has the same direction and the same wavefront as the transmitted signal beam. These two beams interfere on a photodiode what allows the phase demodulation. An external applied electric field allows to control the amplitude and the phase of the index grating compared to the illumination grating given by the complex nature of the photorefractive gain in amplitude $\gamma=\gamma^{\prime}+\mathrm{i} \gamma^{\prime \prime}$, where $\gamma^{\prime}$ is the real part and $\gamma^{\prime \prime}$ the imaginary part of $\gamma$. In all the experiments, the pump to signal beam intensity ratio is much greater than one, what allows to be in the undepleted pump approximation.

${ }^{*}$ Corresponding author: tel: +33 1693588 91; fax: +33 1693587 00; e-mail: sebastien.derossi@iota.u-psud.fr 

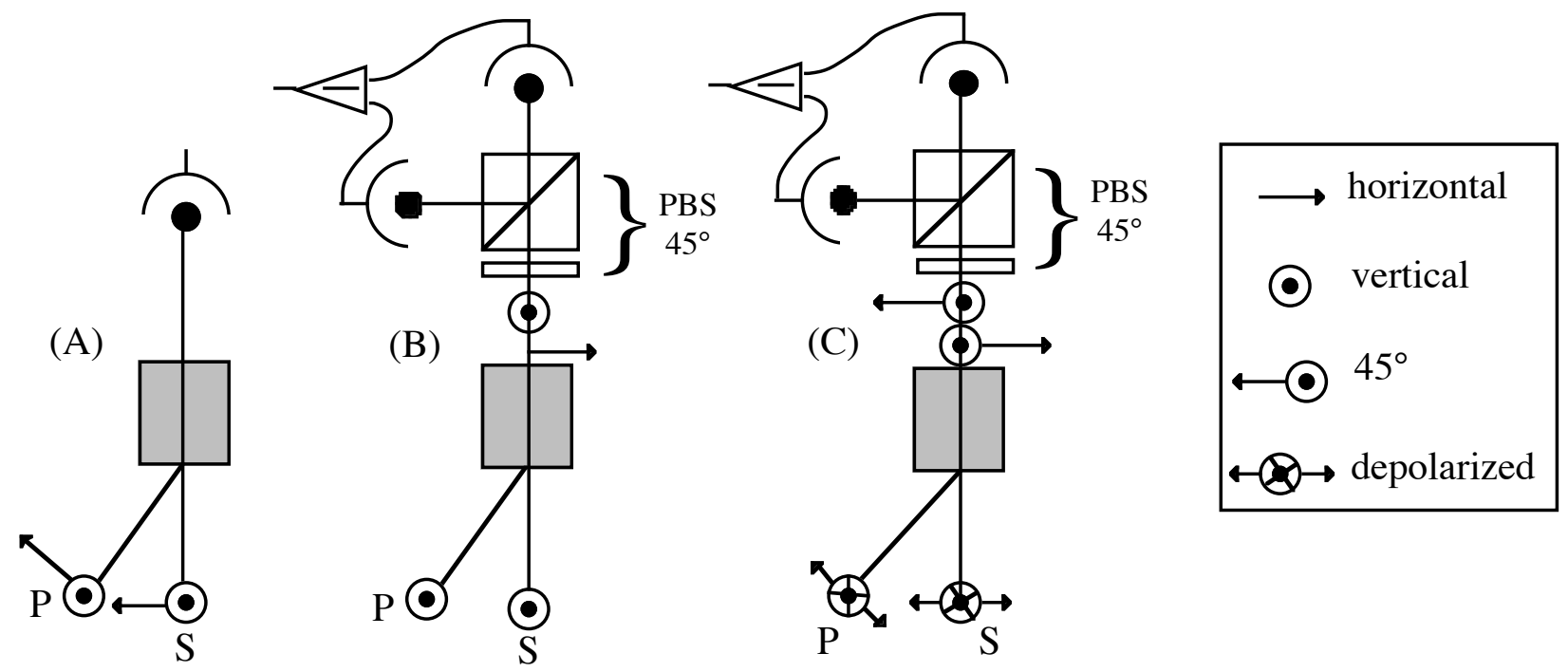

Fig. 1 : Implemented architectures, (A) direct detection, (B) anisotropic diffraction; (C) depolarized configuration. Both beams enter by the (110) faces and the grating vector is along ( 110 .

With polarized beams, two configurations are possible, the direct detection and the anisotropic diffraction ones (fig.1). In the direct detection [1,2] both waves are polarized along the Pockels eigenaxes of the crystal (here at $\pm 45^{\circ}$ ). Local oscillator and signal beam have the same polarization. We detect directly the demodulation with a photodiode. Linear detection is possible only if the imaginary part $\gamma^{\prime \prime}$ of photorefractive gain $\gamma$ is not null, what is obtained with an external field. In the anisotropic diffraction [1] both waves are polarized along (001). The local oscillator is horizontally polarized. If we insert a $45^{\circ}$ polarizer after the crystal, each detector receives the eigenwaves. If we make the difference between the signal of each detector, we have here also a linear detection for $\gamma^{\prime \prime} \neq 0$.

A third solution consists in working with depolarized beams [3]. Both waves record two decorrelated holograms of $90^{\circ}$ polarized images along the eigenaxes (here at $\pm 45^{\circ}$ ). We analyze them independently with a polarizer beam splitter oriented along the eigenaxes. Each wave has a local oscillator and a transmitted signal in phase quadrature thanks to the external field. Contrary to the anistropic diffraction the polarized beams along the different eigenaxes have not the same wavefront and can not interfere together. We thus have two decorrelated wave mixing process in a direct detection configuration with a gain reduced by a factor 2 . By making the difference of the two photodiode signals, we obtain again a linear detection for $\gamma^{\prime \prime} \neq 0$.

To compare our results we use the relative detection limit $\delta_{\text {rel }}$. It is the ratio between the smallest displacement amplitude $\delta$ measured by our sensor (signal to noise ratio of one with an $1 \mathrm{~W}$ power polarized wave incident on the crystal and a bandwidth of $1 \mathrm{~Hz}$ ) and the optimal detection limit of an optical technique $\delta_{\text {opt }}$ :

$$
\delta_{\mathrm{opt}}=\frac{\lambda}{4 \pi} \sqrt{\frac{\mathrm{h}_{v}}{2 \eta}}
$$

$\lambda$ is the wavelength of the laser, $h v$ its energy and $\eta$ the quantum efficiency of the detector. For our three architectures we obtain :

$$
\delta_{r \text { el }}^{\text {dir }}=\frac{e^{\frac{\alpha x}{2}}}{\sin \left(\gamma^{\prime \prime} x\right)} ; \delta_{r \text { el }}^{\text {ani }}=e^{\frac{\alpha x}{2}} \frac{\sqrt{1+||^{2} x^{2}}}{\gamma^{\prime \prime} x} ; \delta_{r e l}^{\text {dep }}=\frac{e^{\frac{\alpha x}{2}}}{\sqrt{2}} \frac{\sqrt{1+\operatorname{th}^{2}\left(\frac{\gamma^{\prime} x}{2}\right)}}{\sin \left(\frac{\gamma^{\prime \prime} x}{2}\right)}
$$

Where $\mathrm{x}$ is the thickness of the crystal along the wave propagation direction, $\alpha$ the absorption.

\section{Photorefractive crystals and measurement of the screening factor}


The photorefractive effect in semiconductors as CdZnTe:V and InP:Fe is now well-known. For the CdZnTe we use one trap model with an electron-hole competition [4]. For InP the photoconduction is governed by holes with an electron-hole competition thermally induced from the excited state of $\mathrm{Fe}^{2+}$ [5]. From photorefractive gain measurements as a function of the grating spacing in diffusion regime, we know all the essential parameters for InP and CdTe photorefractive models. Nevertheless one problem subsists because of inhomogeneities of the crystal and nonuniformity of the pump beam illumination. The applied electric field is screened and the real field $\mathrm{E}_{\mathrm{O}}$ seen by the signal beam is not known. We define the screening factor $\mathrm{c}_{1}$ as :

$$
\mathrm{E}_{\mathrm{o}}=\mathrm{E}_{\mathrm{app}} \mathrm{C}_{1}
$$

Where Eapp $=V_{o} / d$ with $V_{o}$ the applied voltage on crystal and $d$ the inter-electrode spacing.

Determination of $c_{1}$ is obtained by a Pockels effect measurement (see fig. 2). We measure the signal beam transmission between crossed polarizers as a function of the applied field. The experiments have been performed with the same configuration of illumination than that of the ultrasonic detection system. To avoid having a parasite grating, pump and signal polarizations are crossed. We show easily that in our configuration, the transmitted signal $\mathrm{I}_{\mathrm{t}}$ is :

$$
\mathrm{I}_{\mathrm{t}}=\mathrm{I}_{\mathrm{o}} \sin ^{2}\left(\frac{\pi \cdot \mathrm{x}}{\lambda} \mathrm{n}_{\mathrm{o}}^{3} \cdot \mathrm{r}_{4 \mathrm{r}} \mathrm{E}_{\text {app }} \mathrm{C}_{1}\right)
$$

An example of transmission curve is presented in fig. 2.

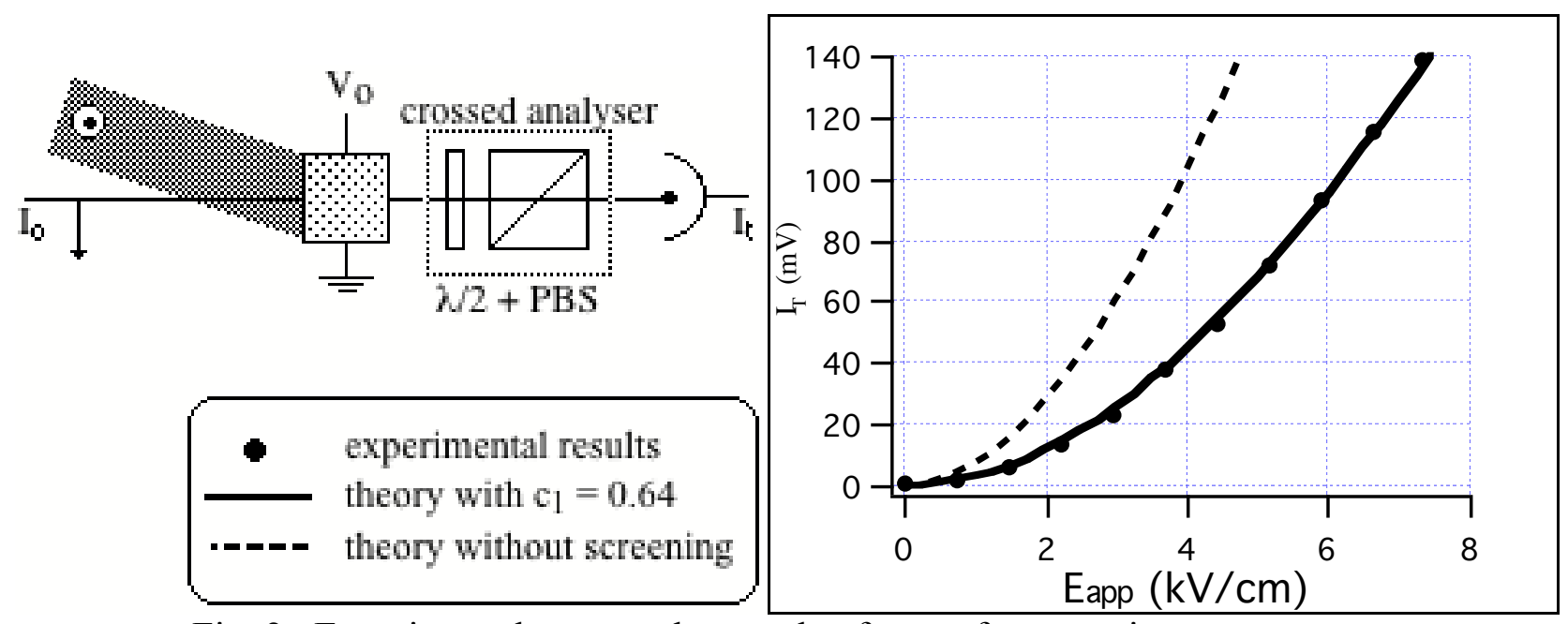

Fig. 2 : Experimental setup and example of curve for screening measurement.

\section{Experimental setup for detection sensitivity measurement}

Beams are obtained from a $600 \mathrm{~mW}$ CW single mode diode pumped Nd:YAG laser emitting at $1.06 \mu \mathrm{m}$. The signal beam is phase modulated with a Pockels cell. The phase modulation is sinusoidal with amplitude of $20 \mathrm{mrad}$ and frequency $80 \mathrm{kHz}$. A multimode fiber module is inserted on each arm to have speckle depolarized beams. Furthermore the pump beam is expanded to cover fully the crystal to minimize the screening of the field and allow a good covering of the signal beam. Illumination is about $150 \mathrm{~mW} / \mathrm{cm}^{2}$. A polarization module polarizes again the beams after the fiber for the direct detection or the anisotropic diffraction configurations. The typical grating spacing is between 3 and $4.5 \mu \mathrm{m}$. The external field is applied to silver painted electrodes during 50 ms. Detectors are InGaAs photodiodes with quantum efficiency $\eta=0.76$.

\section{Detection sensitivity : experimental results}


Measurement presented here (see fig.3) are obtained from a speckled signal beam with similar results obtained from plane signal beam.

For the InP crystal, the screening factor is 0.64 with a plane pump beam. The agreement with the theory is good in the direct detection and anistropic diffraction. We have not been able to work with a speckle pump beam. This is explained as follow. The dark conductivity of InP is greater than the photoconductivity in the some place in the crystal because of the granular nature of the pump beam and the gain hardly saturates regarding the incident intensity. This problem disappears in the pulsed regime.

For CdZnTe we can work with a speckled pump beam without any problem. The curves have been realized with a speckled pump and signal beams. Results are identical for both planes waves or one plane wave and the other speckled. The screening factor is 0.6. We obtain a good agreement with theory if we add a supplementary screening factor $c_{2}=0.5$. This complementary screening factor $c_{2}$ is identical whatever the architecture and the wavefront structure of the beams (plane or speckle wave). It has been observed in other tested CdZnTe crystals. Work is currently in progress to identify the origin of this supplementary screening factor.
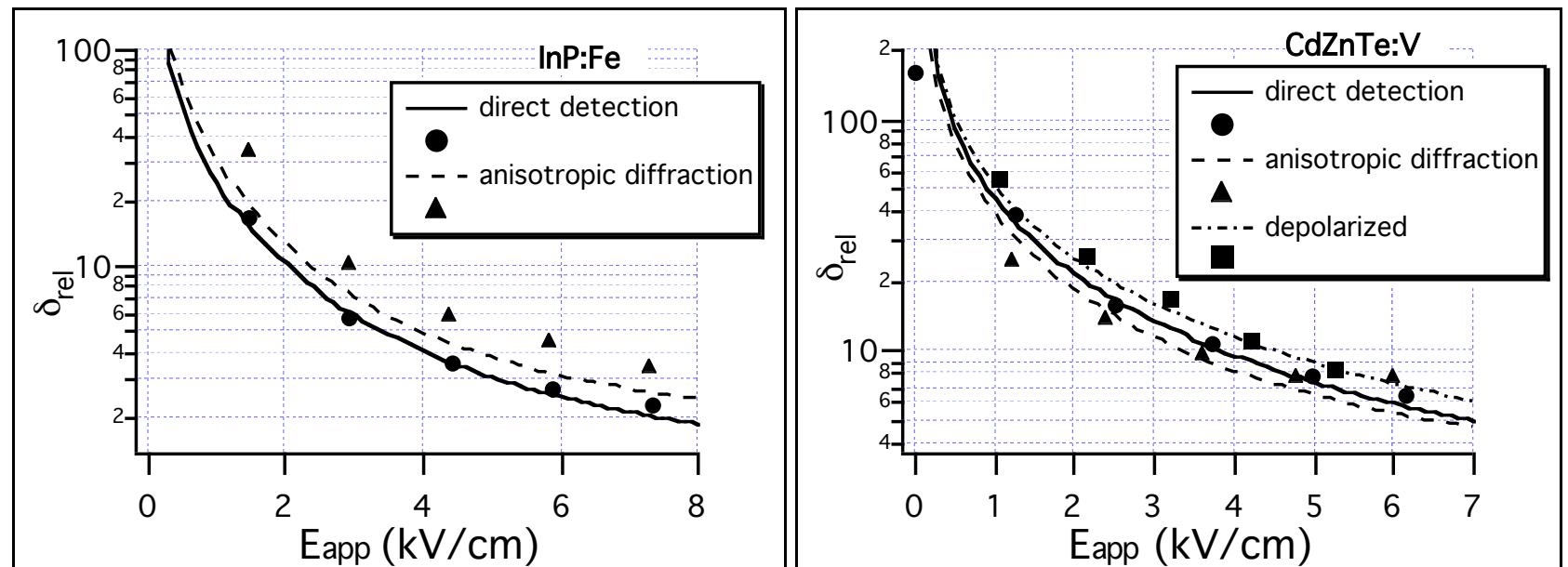

Fig. 3 : Evolution of the relative detection limits as a function of the applied field (markers : experimental measures / lines : theoretical curves).

\section{Conclusion}

The study of various architectures of an ultrasonic sensor allows us to valid theoretical TWM model in presence of a phase modulated signal in photorefractive crystals as InP:Fe and CdZnTe:V. We can now predict the detection sensitivity of each configuration and choose the optimal one. Problems have been emphasized. For CdZnTe the results are less good than expected due to the presence of a supplementary screening factor. We have to understand this drop in performances to operate the sensor at best with CdZnTe.

\section{References}

[1] Ph. Delaye, A. Blouin, D. Drolet, J.P. Monchalin, L.A. de Montmorillon, G. Roosen, J. Opt. Soc. Am. B, 14, 1723 (1997).

[2] L.A. de Montmorillon, I. Baggio, Ph. Delaye, J.C. Launay, G. Roosen, Opt. Comm., 129, 293 (1996)

[3] Ph. Delaye, A. Blouin, D. Drolet, J.P. Monchalin, L.A. de Montmorillon, G. Roosen, Appl. Phys. Lett., 74, 3087 (1999).

[4] F.P. Strohkendl, J.M.C. Jonathan, R.W. Hellwarth, Opt. Lett., 11, 312 (1986).

[5] Ph. Delaye, P.U. Halter, G. Roosen, J. Opt. Soc. Am. B, 7, 2268 (1990). 